\title{
Quasi-normal modes of Schwarzschild-de Sitter black holes
}

\author{
A. Zhidenko \\ Department of Physics, Dniepropetrovsk National University \\ St. Naukova 13, Dniepropetrovsk 49050, Ukraine
}

\begin{abstract}
The low-laying frequencies of characteristic quasi-normal modes (QNM) of Schwarzschild-de Sitter (SdS) black holes have been calculated for fields of different spin using the sixth-order WKB approximation and the approximation by the Pöshl-Teller potential. The well-known asymptotic formula for large $l$ is generalized here on a case of the Schwarzschild-de Sitter black hole. In the limit of the near extreme $\Lambda$ term the results given by both methods are in a very good agreement, and in this limit fields of different spin decay with the same rate.
\end{abstract}




\section{Introduction}

Within general relativity it is well-known that there are three stages to the dynamical evolution of field perturbation on a black hole background: the initial outburst from the source of perturbation, the damping (quasi-normal) oscillations and asymptotic tails at very late time. The evolution significantly depends on the asymptotic behavior of the background: for different values of cosmological constant $\Lambda$ there are three types of behavior depending on the sign of $\Lambda$. Thus, the remarkable fact is that while for asymptotically flat backgrounds we have inverse power law tails, for asymptotically Anti-de Sitter background it is exponential low that governs the decay of a field at all times. The same exponential decay at very late times was observed by Brady et al [1] for asymptotically de Sitter background.

Apart from original interest coming from possibility to observe quasi-normal ringing with the help of gravitational wave detectors (see, e.g., [2] for review), the quasi-normal modes bring now a lot of interest in different contexts: in Anti-de Sitter(de Sitter)/Conformal Field Theory correspondence $[3,4,5]$, when considering thermodynamic properties of black holes in loop quantum gravity [6], in the context of possible connection with critical collapse [3, 7].

The gravitational QNMs of Schwarzschild-de Sitter black hole were considered in [8, 9, 5]. Recently, asymptotic values of QNMs were studied for nearly extremal Schwarzschild-de Sitter black hole and it was shown that in the near extremal limit the effective potential of the wave equation reduces to the Pöshl-Teller potential, and it is expected therefore the Pöshl-Teller method provides the best accuracy near the extremal value of $\Lambda$ [11].

QNMs corresponding to decay of fields of different spin are extensively studied for Schwarzschild and Schwarzschild-anti de Sitter backgrounds. At the same time there is lack of such study of the Schwarzschild-de Sitter case. This motivated us to calculate QNMs of scalar, electromagnetic, gravitational and Dirac fields for Schwarzschild-de Sitter black hole. We also compare the results found here with those obtained recently in $[10,11]$.

In section 2 we give the perturbation equations to describe fields of different spin in SdS background. Section 3 presents comparison of QNM frequencies obtained through sixth order WKB formula and approximation by the Pöshl-Teller potential.

There was shown that the frequencies of the massless Dirac field perturbations [10] are the same for both chiralities in SdS background as well. Near the extremal value of $\Lambda$ the sixth order WKB formula gives the values which are very close to those obtained within the Pöshl-Teller potential approximation. This agrees with recent work of Cardoso and Lemos [11].

\section{Perturbation equations and methods}

It is well known that the Schwarzschild-de Sitter black hole of mass $M$ is described by the metric:

$$
d s^{2}=f(r) d t^{2}-\frac{d r^{2}}{f(r)}-r^{2} d \sigma^{2} ; \quad f(r)=1-\frac{2 M}{r}-\Lambda \frac{r^{2}}{3}, \quad d \sigma^{2}=d \theta^{2}+\sin ^{2} \theta d \phi^{2} .
$$

A perturbation equation on this background can be reduced to the Schrödinger wave-like equations:

$$
\left(\frac{d^{2}}{d r^{* 2}}+\omega^{2}-V\left(r^{*}\right)\right) \Psi\left(r^{*}\right)=0,
$$


where we assume exponential dependence of $\Psi$ on time $\left(\sim e^{-i \omega t}\right), \omega=\omega_{R e}-i \omega_{I m}, r^{*}$ is the "tortoise" coordinate:

$$
d r^{*}=\frac{d r}{f(r)}
$$

Under the positive real part QNMs, by definition, satisfy the following boundary conditions:

$$
\Psi\left(r^{*}\right) \sim C_{ \pm} \exp \left( \pm i \omega r^{*}\right), \quad r \longrightarrow \pm \infty
$$

which correspond to purely out-going waves at infinity and in-going waves at the horizon.

The corresponding perturbation equations can be rewritten in form (2) with potentials

$$
\begin{aligned}
V_{s} & =f(r)\left(\frac{l(l+1)}{r^{2}}+\frac{1}{r} f^{\prime}(r)\right) ; \\
V_{\text {el }} & =f(r) \frac{l(l+1)}{r^{2}} ; \\
V_{\text {odd }} & =f(r)\left(\frac{l(l+1)}{r^{2}}-\frac{6 M}{r^{3}}\right) ; \\
V_{\text {even }} & =\frac{2 f(r)}{r^{3}} \frac{9 M^{3}+3 c^{2} M r^{2}+c^{2}(1+c) r^{3}+3 M^{2}\left(3 c r-\Lambda r^{3}\right)}{(3 M+c r)^{2}}, \quad c=\frac{l(l+1)}{2}-1 ; \\
V_{d} & =\frac{\kappa f^{1 / 2}(r)}{r^{2}}\left(\kappa f^{1 / 2}(r) \pm\left(\frac{3 M}{r}-1\right)\right) ;
\end{aligned}
$$

for massless scalar, electromagnetic, gravitational (odd end even parities) and Dirac fields respectively (see, e.g., [12] and references therein), where $l$ is the multipole number, $\kappa=l+1$ for $(+)$ sign and $\kappa=l$ for $(-)$ sign in (9). All the potentials vanish at the event horizon and the cosmological horizon, having a peak near the event horizon. It allows to use the Pöshl-Teller potential approximation as well as WKB method.

The WKB approach is based on the analogy with the problem of waves scattering near the peak of the potential barrier $V\left(r^{*}\right)$ in quantum mechanics, where $\omega^{2}$ plays a role of energy. The approach was used by Schutz and Will [13], developed to the third order by Iyer and Will [14], and recently developed by Konoplya to the sixth order beyond the eikonal approximation [15]. The result has the form:

$$
i \frac{\omega^{2}-V_{0}}{\sqrt{-2 V_{0}^{\prime \prime}}}-\Lambda_{2}-\Lambda_{3}-\Lambda_{4}-\Lambda_{5}-\Lambda_{6}=n+\frac{1}{2},
$$

where $V_{0}$ is the height and $V_{0}^{\prime \prime}$ is the second derivative with respect to the tortoise coordinate of the potential at the maximum. $\Lambda_{2}$ and $\Lambda_{3}$ can be found in [14], $\Lambda_{4}, \Lambda_{5}$ and $\Lambda_{6}$ are presented in [15]; the corrections depend on the value of the potential and higher derivatives of it at the maximum.

Generally, WKB method was effectively used for finding quasi-normal frequencies in a lot of papers [16]. Comparing the values calculated by the different order WKB formula one can judge about the convergence to the some unknown accurate result as the WKB order increasing. We expect that the possible error is less then the difference between the sixth and the fifth order WKB values ${ }^{1}$. Note,

\footnotetext{
${ }^{1}$ The accuracy of values are to take as a half of the number last order (e. g. 0.125 means $0.125 \pm 0.003$ ), but it is much better for most of them.
} 
that standard machine precision in MATHEMATICA gives large errors due to numerical truncations. To exclude such "noises" we had to hold 40 digits in all intermediate values.

For comparison we will use the semi-analytical method for calculations proposed by B. Mashhoon [18] who used Pöshl-Teller approximate potential

$$
V_{P T}=\frac{V_{0}}{\cosh ^{2}\left(r^{*} / b\right)} .
$$

It contains two free parameters $\left(V_{0}\right.$ and $\left.b\right)$ which are used to fit the height and the second derivative of the potential $V\left(r^{*}\right)$ at the maximum [18].

The quasi-normal modes of the Pöshl-Teller potential can be evaluated analytically:

$$
\omega=\frac{1}{b}\left(\sqrt{V_{0} b^{2}-\frac{1}{4}}-\left(n+\frac{1}{2}\right) i\right) .
$$

\section{QNMs of SdS black holes}

Both methods give best result for low overtone number but it is known that the higher modes give larger values of the imaginary part of frequencies [2]. Therefore these modes decay faster then lowlying ones that are more interesting for us. So we calculated some first quasi-normal mode frequencies for massless scalar, electromagnetic and gravitational and Dirac fields using both of the methods. The results are presented below (all values are measured in the black hole mass (M) units).

QNMs of massless scalar field: $l=1, n=0$

\begin{tabular}{|r|r|r|}
\hline$\Lambda$ & $\omega(\mathrm{WKB})$ & $\omega(\mathrm{P}-\mathrm{T})$ \\
\hline 0 & $0.2929-0.0978 i$ & $0.299-0.101 i$ \\
0.02 & $0.2603-0.0911 i$ & $0.263-0.093 i$ \\
0.04 & $0.2247-0.0821 i$ & $0.226-0.083 i$ \\
0.06 & $0.1854-0.0701 i$ & $0.187-0.071 i$ \\
0.08 & $0.1404-0.0542 i$ & $0.141-0.055 i$ \\
0.09 & $0.11392-0.04397 i$ & $0.1147-0.0443 i$ \\
0.10 & $0.08156-0.03121 i$ & $0.0819-0.0315 i$ \\
0.11 & $0.02549-0.00965 i$ & $0.02550-0.00967 i$ \\
\hline
\end{tabular}

QNMs of massless scalar field: $l=2, n=1$

\begin{tabular}{|r|r|r|}
\hline$\Lambda$ & $\omega(\mathrm{WKB})$ & $\omega(\mathrm{P}-\mathrm{T})$ \\
\hline 0 & $0.46385-0.29563 i$ & $0.487-0.294 i$ \\
0.02 & $0.42084-0.26862 i$ & $0.437-0.268 i$ \\
0.04 & $0.37165-0.23796 i$ & $0.382-0.238 i$ \\
0.06 & $0.31422-0.20163 i$ & $0.321-0.202 i$ \\
0.08 & $0.24426-0.15627 i$ & $0.248-0.156 i$ \\
0.09 & $0.20098-0.12794 i$ & $0.2033-0.1282 i$ \\
0.10 & $0.14576-0.09212 i$ & $0.1468-0.0923 i$ \\
0.11 & $0.04614-0.02889 i$ & $0.04617-0.02890 i$ \\
\hline
\end{tabular}

QNMs of massless scalar field: $l=2, n=0$

\begin{tabular}{|r|r|r|}
\hline$\Lambda$ & $\omega(\mathrm{WKB})$ & $\omega(\mathrm{P}-\mathrm{T})$ \\
\hline 0 & $0.48364-0.09677 i$ & $0.487-0.098 i$ \\
0.02 & $0.43461-0.08858 i$ & $0.437-0.089 i$ \\
0.04 & $0.38078-0.07876 i$ & $0.382-0.079 i$ \\
0.06 & $0.32002-0.06685 i$ & $0.321-0.067 i$ \\
0.08 & $0.24747-0.05197 i$ & $0.248-0.052 i$ \\
0.09 & $0.20296-0.04256 i$ & $0.2033-0.0427 i$ \\
0.10 & $0.14661-0.03069 i$ & $0.1468-0.0308 i$ \\
0.11 & $0.04617-0.00963 i$ & $0.04617-0.00963 i$ \\
\hline
\end{tabular}

QNMs of electromagnetic field: $l=1, n=0$

\begin{tabular}{|r|r|r|}
\hline$\Lambda$ & $\omega(\mathrm{WKB})$ & $\omega(\mathrm{P}-\mathrm{T})$ \\
\hline 0 & $0.2482-0.0926 i$ & $0.255-0.096 i$ \\
0.02 & $0.2259-0.0842 i$ & $0.231-0.087 i$ \\
0.04 & $0.2006-0.0748 i$ & $0.204-0.077 i$ \\
0.06 & $0.1709-0.0639 i$ & $0.173-0.065 i$ \\
0.08 & $0.1339-0.0502 i$ & $0.135-0.051 i$ \\
0.09 & $0.11053-0.04156 i$ & $0.1110-0.0419 i$ \\
0.10 & $0.08035-0.03028 i$ & $0.0805-0.0304 i$ \\
0.11 & $0.02545-0.00962 i$ & $0.02546-0.00962 i$ \\
\hline
\end{tabular}


QNMs of electromagnetic field: $l=2, n=0$

\begin{tabular}{|r|r|r|}
\hline$\Lambda$ & $\omega(\mathrm{WKB})$ & $\omega(\mathrm{P}-\mathrm{T})$ \\
\hline 0 & $0.45759-0.09501 i$ & $0.461-0.096 i$ \\
0.02 & $0.41502-0.08615 i$ & $0.418-0.087 i$ \\
0.04 & $0.36723-0.07624 i$ & $0.369-0.077 i$ \\
0.06 & $0.31182-0.06478 i$ & $0.313-0.065 i$ \\
0.08 & $0.24365-0.05067 i$ & $0.244-0.051 i$ \\
0.09 & $0.20085-0.04180 i$ & $0.2012-0.0419 i$ \\
0.10 & $0.14582-0.03037 i$ & $0.1459-0.0304 i$ \\
0.11 & $0.04614-0.00962 i$ & $0.04615-0.00962 i$ \\
\hline
\end{tabular}

QNMs of electromagnetic field: $l=2, n=1$

\begin{tabular}{|r|r|r|}
\hline$\Lambda$ & $\omega(\mathrm{WKB})$ & $\omega(\mathrm{P}-\mathrm{T})$ \\
\hline 0 & $0.43653-0.29073 i$ & $0.461-0.289 i$ \\
0.02 & $0.39900-0.26202 i$ & $0.418-0.261 i$ \\
0.04 & $0.35602-0.23065 i$ & $0.369-0.231 i$ \\
0.06 & $0.30498-0.19516 i$ & $0.313-0.196 i$ \\
0.08 & $0.24046-0.15223 i$ & $0.244-0.153 i$ \\
0.09 & $0.19907-0.12549 i$ & $0.2012-0.1258 i$ \\
0.10 & $0.14515-0.09114 i$ & $0.1459-0.0913 i$ \\
0.11 & $0.04612-0.02886 i$ & $0.04615-0.02887 i$ \\
\hline
\end{tabular}

QNMs of gravitational field: $l=2, n=0$

\begin{tabular}{|l|r|r|r|r|}
\hline$\Lambda$ & $\omega(\mathrm{WKB})(\mathrm{odd})$ & $\omega(\mathrm{P}-\mathrm{T})$ (odd) & $\omega$ (WKB) $($ even $)$ & $\omega(\mathrm{P}-\mathrm{T})($ even $)$ \\
\hline 0 & $0.3736-0.0889 i$ & $0.378-0.091 i$ & $0.3737-0.0889 i$ & $0.378-0.091 i$ \\
0.02 & $0.3384-0.0817 i$ & $0.342-0.083 i$ & $0.3384-0.0817 i$ & $0.342-0.083 i$ \\
0.04 & $0.2989-0.0733 i$ & $0.301-0.074 i$ & $0.2989-0.0733 i$ & $0.301-0.074 i$ \\
0.06 & $0.2533-0.0630 i$ & $0.254-0.064 i$ & $0.2533-0.0630 i$ & $0.254-0.064 i$ \\
0.08 & $0.1975-0.0499 i$ & $0.198-0.050 i$ & $0.1975-0.0499 i$ & $0.198-0.050 i$ \\
0.09 & $0.16261-0.04136 i$ & $0.1629-0.0415 i$ & $0.16261-0.04137 i$ & $0.1629-0.0415 i$ \\
0.10 & $0.11792-0.03021 i$ & $0.1180-0.0303 i$ & $0.11792-0.03021 i$ & $0.1180-0.0303 i$ \\
0.11 & $0.03727-0.00962 i$ & $0.03727-0.00962 i$ & $0.03727-0.00962 i$ & $0.03727-0.00962 i$ \\
\hline
\end{tabular}

QNMs of gravitational field: $l=2, n=1$

\begin{tabular}{|r|r|r|r|r|}
\hline$\Lambda$ & $\omega($ WKB $)$ (odd) & $\omega(\mathrm{P}-\mathrm{T})$ (odd) & $\omega$ (WKB) (even) & $\omega(\mathrm{P}-\mathrm{T})$ (even) \\
\hline 0 & $0.3463-0.2735 i$ & $0.378-0.272 i$ & $0.3467-0.2739 i$ & $0.378-0.272 i$ \\
0.02 & $0.3185-0.2488 i$ & $0.342-0.249 i$ & $0.3188-0.2491 i$ & $0.342-0.249 i$ \\
0.04 & $0.2858-0.2215 i$ & $0.301-0.223 i$ & $0.2858-0.2217 i$ & $0.301-0.223 i$ \\
0.06 & $0.2457-0.1896 i$ & $0.254-0.191 i$ & $0.2457-0.1898 i$ & $0.254-0.191 i$ \\
0.08 & $0.1941-0.1497 i$ & $0.198-0.150 i$ & $0.1941-0.1498 i$ & $0.198-0.150 i$ \\
0.09 & $0.16079-0.12412 i$ & $0.1629-0.1246 i$ & $0.16079-0.12415 i$ & $0.1629-0.1246 i$ \\
0.10 & $0.11724-0.09063 i$ & $0.1180-0.0908 i$ & $0.11724-0.09064 i$ & $0.1180-0.0908 i$ \\
0.11 & $0.03725-0.02885 i$ & $0.03727-0.02885 i$ & $0.03725-0.02885 i$ & $0.03727-0.02885 i$ \\
\hline
\end{tabular}


QNMs of gravitational field: $l=3, n=0$

\begin{tabular}{|r|r|r|r|r|}
\hline$\Lambda$ & $\omega$ (WKB) (odd) & $\omega(\mathrm{P}-\mathrm{T})$ (odd) & $\omega$ (WKB) (even) & $\omega$ (P-T) (even) \\
\hline 0 & $0.599443-0.092703 i$ & $0.602-0.093 i$ & $0.599443-0.092703 i$ & $0.602-0.093 i$ \\
0.02 & $0.543115-0.084495 i$ & $0.545-0.085 i$ & $0.543115-0.084496 i$ & $0.545-0.085 i$ \\
0.04 & $0.480058-0.075146 i$ & $0.481-0.076 i$ & $0.480058-0.075146 i$ & $0.481-0.076 i$ \\
0.06 & $0.407175-0.064140 i$ & $0.408-0.064 i$ & $0.407175-0.064140 i$ & $0.408-0.064 i$ \\
0.08 & $0.317805-0.050382 i$ & $0.318-0.051 i$ & $0.317804-0.050382 i$ & $0.318-0.051 i$ \\
0.09 & $0.261841-0.041644 i$ & $0.2621-0.0417 i$ & $0.261843-0.041643 i$ & $0.2621-0.0417 i$ \\
0.10 & $0.189994-0.030314 i$ & $0.1901-0.0303 i$ & $0.189994-0.030314 i$ & $0.1901-0.0303 i$ \\
0.11 & $0.060091-0.009619 i$ & $0.06009-0.00962 i$ & $0.060091-0.009619 i$ & $0.06009-0.00962 i$ \\
\hline
\end{tabular}

QNMs of gravitational field: $l=3, n=1$

\begin{tabular}{|r|r|r|r|r|}
\hline$\Lambda$ & $\omega$ (WKB) (odd) & $\omega$ (P-T) (odd) & $\omega$ (WKB) (even) & $\omega$ (P-T) (even) \\
\hline 0 & $0.58264-0.28129 i$ & $0.602-0.280 i$ & $0.58264-0.28129 i$ & $0.602-0.280 i$ \\
0.02 & $0.53074-0.25536 i$ & $0.545-0.255 i$ & $0.53074-0.25536 i$ & $0.545-0.255 i$ \\
0.04 & $0.47166-0.22639 i$ & $0.481-0.227 i$ & $0.47166-0.22639 i$ & $0.481-0.227 i$ \\
0.06 & $0.40217-0.19281 i$ & $0.408-0.193 i$ & $0.40217-0.19281 i$ & $0.408-0.193 i$ \\
0.08 & $0.31550-0.15125 i$ & $0.318-0.152 i$ & $0.31549-0.15125 i$ & $0.318-0.152 i$ \\
0.09 & $0.26056-0.12498 i$ & $0.2621-0.1251 i$ & $0.26057-0.12497 i$ & $0.2621-0.1251 i$ \\
0.10 & $0.18952-0.09095 i$ & $0.1901-0.0910 i$ & $0.18952-0.09095 i$ & $0.1901-0.0910 i$ \\
0.11 & $0.06008-0.02886 i$ & $0.06009-0.02886 i$ & $0.06008-0.02886 i$ & $0.06009-0.02886 i$ \\
\hline
\end{tabular}

QNMs of massless Dirac field: $\kappa=1, n=0$

\begin{tabular}{|r|r|r|r|r|}
\hline$\Lambda$ & $\omega(\mathrm{WKB})(-)$ & $\omega(\mathrm{P}-\mathrm{T})(-)$ & $\omega(\mathrm{WKB})(+)$ & $\omega(\mathrm{P}-\mathrm{T})(+)$ \\
\hline 0 & $0.183-0.097 i$ & $0.183-0.098 i$ & $0.183-0.095 i$ & $0.189-0.105 i$ \\
0.02 & $0.167-0.087 i$ & $0.166-0.088 i$ & $0.167-0.085 i$ & $0.171-0.094 i$ \\
0.04 & $0.149-0.077 i$ & $0.147-0.077 i$ & $0.149-0.075 i$ & $0.151-0.082 i$ \\
0.06 & $0.128-0.069 i$ & $0.127-0.065 i$ & $0.128-0.064 i$ & $0.128-0.069 i$ \\
0.08 & $0.1005-0.0507 i$ & $0.0985-0.0505 i$ & $0.1006-0.0501 i$ & $0.1001-0.0529 i$ \\
0.09 & $0.0832-0.0418 i$ & $0.0813-0.0415 i$ & $0.0833-0.0414 i$ & $0.0824-0.0432 i$ \\
0.10 & $0.0606-0.0304 i$ & $0.0592-0.0301 i$ & $0.0606-0.0303 i$ & $0.0597-0.0311 i$ \\
0.11 & $0.0192-0.0096 i$ & $0.0188-0.0096 i$ & $0.0192-0.0096 i$ & $0.0189-0.0097 i$ \\
\hline
\end{tabular}


QNMs of massless Dirac field: $\kappa=2, n=0$

\begin{tabular}{|r|r|r|r|r|}
\hline$\Lambda$ & $\omega(\mathrm{WKB})(-)$ & $\omega(\mathrm{P}-\mathrm{T})(-)$ & $\omega(\mathrm{WKB})(+)$ & $\omega(\mathrm{P}-\mathrm{T})(+)$ \\
\hline 0 & $0.38004-0.09642 i$ & $0.382-0.097 i$ & $0.38007-0.09637 i$ & $0.386-0.099 i$ \\
0.02 & $0.34491-0.08715 i$ & $0.346-0.088 i$ & $0.34493-0.08712 i$ & $0.349-0.089 i$ \\
0.04 & $0.30542-0.07690 i$ & $0.306-0.077 i$ & $0.30543-0.07688 i$ & $0.308-0.079 i$ \\
0.06 & $0.25953-0.06516 i$ & $0.260-0.065 i$ & $0.25954-0.06515 i$ & $0.261-0.066 i$ \\
0.08 & $0.20296-0.05085 i$ & $0.203-0.051 i$ & $0.20296-0.05084 i$ & $0.204-0.052 i$ \\
0.09 & $0.16738-0.04190 i$ & $0.167-0.0418 i$ & $0.16738-0.04190 i$ & $0.168-0.0423 i$ \\
0.10 & $0.12157-0.03041 i$ & $0.121-0.0304 i$ & $0.12157-0.03041 i$ & $0.122-0.0305 i$ \\
0.11 & $0.03849-0.00962 i$ & $0.0384-0.0096 i$ & $0.03849-0.00962 i$ & $0.0384-0.0096 i$ \\
\hline
\end{tabular}

The relative error of the WKB method depends significantly on a field under consideration. Comparison of the sixth and fifth WKB order let us to conclude that the worst WKB convergence gives the Dirac field, where the expected error reaches $1 \%$ in the sixth WKB order. The results calculated in [10] by using the third WKB order differ from the sixth order values up to 4\%. Thus, the possible relative error of the third order WKB results may exceed $4 \%$.

We can also evaluate the relative error order for gravitational modes by comparison QNMs of odd and even perturbations. Because the potentials can be expressed in the form

$$
V_{\text {odd }}=W_{g}^{2}+\frac{d W_{g}}{d r^{*}}+\beta ; \quad V_{\text {even }}=W_{g}^{2}-\frac{d W_{g}}{d r^{*}}+\beta
$$

where

$$
W_{g}=\frac{2 M}{r^{2}}-\frac{3+2 c}{3 r}+\frac{3 c^{2}+2 c^{3}-9 \Lambda M^{2}}{3 c(3 M+c r)}-\frac{1}{3 M}\left(c^{2}+c-\frac{3 \Lambda M^{2}}{c}\right) ; \quad \beta=-\frac{c^{2}(c+1)^{2}}{9 M^{2}} ;
$$

any solution for even parity can be found from the solution for odd parity [17]:

$$
\Psi_{\text {even }}\left(r^{*}\right)=q\left(W_{g}\left(r^{*}\right)-\frac{d}{d r^{*}}\right) \Psi_{o d d}\left(r^{*}\right) ; \quad q=\text { const } .
$$

Obviously, if a solution $\Psi_{\text {odd }}$ describing quasi-normal oscillations satisfies the boundary conditions (4), the $\Psi_{\text {even }}$ solution satisfies the same ones describing quasi-normal oscillations too.

The same fact [12] takes place for massless Dirac QNMs of opposite chiralities:

$$
V_{d}=W_{d}^{2} \pm \frac{d W_{d}}{d r^{*}} ; \quad W_{d}(r)=\frac{\kappa \sqrt{f(r)}}{r} .
$$

Therefore, the QNM spectrum is the same for odd and even gravitational perturbations and for opposite chirality of Dirac perturbations in SdS background as well as in asymptotically flat universe. We see that the difference between the numerical results does not exceed our expectations.

We can see that the imaginary parts of QNMs, calculated using both sixth-order WKB and Pöshl-Teller approaches, depend on multipole and overtone number and black hole mass only near 
the extremal value of $\Lambda$. This agrees with [11], where the QNMs of near extremal SdS space-time were found:

$$
\omega b=-\left(n+\frac{1}{2}\right) i+\sqrt{l(l+1)-\frac{1}{4}}
$$

for scalar and electromagnetic perturbations,

$$
\omega b=-\left(n+\frac{1}{2}\right) i+\sqrt{(l+2)(l-1)-\frac{1}{4}}
$$

for gravitational perturbations. Here

$$
b=\frac{54 M^{3}}{\left(r_{c}-3 M\right)\left(r_{c}+6 M\right)}, \quad r_{c} \rightarrow 3 M .
$$

Using the same technique, one can find that

$$
\omega b=-\left(n+\frac{1}{2}\right) i+\sqrt{\kappa^{2}-\frac{1}{4}}
$$

for massless Dirac field perturbations. It means in the extremal limit fields of different spin decay with the same rate. Note, that formulae (15), (16), (17) give right results for any overtone number $n$ and may be useful for studying the $n \rightarrow \infty$ limit.

The well-known approximate formula for large multipole number $l$ (or $\kappa$ in (9)) [18, 19]:

$$
\begin{aligned}
\omega & =\frac{1}{3 \sqrt{3} M}\left(l+\frac{1}{2}-\left(n+\frac{1}{2}\right) i\right)+O\left(\frac{1}{l}\right) ; \\
\omega & =\frac{1}{3 \sqrt{3} M}\left(\kappa-\left(n+\frac{1}{2}\right) i\right)+O\left(\frac{1}{\kappa}\right)
\end{aligned}
$$

can be generalized for the case of non-zero $\Lambda$. Making use of the first-order WKB method or the formula of the approximation by the Pöshl-Teller potential one can find for the non-extremal SdS black hole:

$$
\begin{aligned}
& \omega=\frac{\sqrt{1-9 M^{2} \Lambda}}{3 \sqrt{3} M}\left(l+\frac{1}{2}-\left(n+\frac{1}{2}\right) i\right)+O\left(\frac{1}{l}\right) ; \\
& \omega=\frac{\sqrt{1-9 M^{2} \Lambda}}{3 \sqrt{3} M}\left(\kappa-\left(n+\frac{1}{2}\right) i\right)+O\left(\frac{1}{\kappa}\right) .
\end{aligned}
$$

\section{Conclusion}

The QNMs of Schwarzschild-de Sitter black holes for fields of different spin have been calculated. The modes are determined by the black hole mass $M$ and the cosmological constant $\Lambda$ only. The frequencies all have a negative imaginary part, which means that the black hole is stable against these perturbations. The presence of the cosmological constant leads to decrease of the real oscillation frequency and to a slower decay. The interesting problem that was outside our consideration is the search of asymptotic behavior of SdS QNMs at large imaginary part $(n \rightarrow \infty)$ for which other approaches should be explored [20]. 


\section{Acknowledgements}

I would like to thank R. Konoplya for proposing this problem to me and reading the manuscript.

\section{References}

[1] P. Brady, C. Chambers, W. Krivan and P. Laguna, Phys. Rev. D55 7538 (1997);

P. Brady, C. Chambers, W. Laarakkers and E. Poison, Phys. Rev. D60 064003 (1999).

[2] K. Kokkotas and B. Schmidt, Living. Reviews. Relativ. 22 (1999);

H. P. Nollert, Class. Quant. Grav. 16 R159 (1999).

[3] G. T. Horowitz and V. Hubeny, Phys. Rev. D62 024027 (2000).

[4] V. Cardoso and J. P. S. Lemos Phys. Rev. D63 124015 (2001);

J. S. F. Chan and R. B. Mann, Phys. Rev. D55 7546-7562 (1997);

J. S. F. Chan and R. B. Mann, Phys. Rev. D59 064025 (1999);

D. Birmingham, I. Sachs, and S. N. Solodukhin, Phys. Rev. Lett. 88151301 (2002);

D. Birmingham, I. Sachs, and S. N. Solodukhin, Phys. Rev. D67 104026 (2003);

R. A. Konoplya, Phys. Rev. D66 044009 (2002);

R. A. Konoplya, Phys. Rev. D66 084007 (2002);

A. O. Starinets, Phys. Rev. D66 124013 (2002);

V. Cardoso, R. A. Konoplya and J. P. S. Lemos, Phys. Rev. D68 044024 (2003);

E. Abdalla, K. H. C. Castello-Branco and A. Lima-Santos, Phys. Rev. D66 104018 (2002);

R. Aros, C. Martinez, R. Troncoso, and J. Zanelli, Phys. Rev. D67 044014 (2003);

S. Fernando, Gen. Relat. Grav. 36 71-82 (2004).

[5] I. G. Moss and J. P. Norman, Class. Quant. Grav. 192323 (2002).

[6] O. Dreyer, Phys. Rev. Lett. 90081301 (2003);

G. Kunstatter, Phys. Rev. Lett. 90161301 (2003);

L. Motl, Adv. Theor. Math. Phys. 6 1135-1162 (2003).

[7] R.A.Konoplya, Phys. Lett. B550 117 (2002);

W. T. Kim and J. J. Oh, Phys. Lett. B514 155 (2001).

[8] H. Otsuki and T. Futamase, Progress of Theoretical Physics 85771 (1991).

[9] F. Mellor and I. Moss, Phys. Rev. D41 403 (1990).

[10] H. T. Cho, Phys. Rev. D68 024003 (2003).

[11] V. Cardoso and J. P. S. Lemos, Phys. Rev. D67 084020 (2003).

[12] T. Regge and J. A. Wheeler Phys. Rev. 108 1063-1069 (1957);

U. Khanal Phys. Rev. D28 1291 (1983). 
[13] B. F. Schutz and C. M. Will Astrophys. J. 291 L33-L36 (1985).

[14] S. Iyer and C. M. Will, Phys. Rev. D35 3621 (1987).

[15] R. A. Konoplya, Phys. Rev. D68 024018 (2003).

[16] R. A. Konoplya, Gen. Relat. Grav. 34329 (2002);

O. B. Zaslavskii, Phys. Rev. D43 605 (1991);

L. E. Simone and C. M. Will, Class. Quant. Grav. 9963 (1992);

S. Iyer, Phys. Rev. D35 3632 (1987);

K. Kokkotas, and B. F. Schutz, Phys. Rev. D37 3378 (1988);

E. Berti \& K. Kokkotas, Phys. Rev. D67 064020 (2003);

E. Berti \& K. Kokkotas, Phys. Rev. D68 044027 (2003);

E. Berti, V. Cardoso, K. Kokkotas \& Onozawa, Phys. Rev. D68 124018 (2003).

[17] S. Chandrasekhar, in The Mathematical Theory of Black Holes, Oxford University Press, New York (1983).

[18] V. Ferrari, B. Mashhoon Phys. Rev. D30 295-304 (1984).

[19] W. H. Press, Astrophys. J. 170 L105 (1971).

[20] H.-P. Nollert Phys. Rev. D47 5253 (1993).

[21] V. Suneeta Phys. Rev. D68 024020 (2003). 